\title{
Least Squares Estimation of Hydraulic Conductivity from Field Data
}

\author{
K. R. Bailey, $\quad$ B. G. Fitzpatrick ${ }^{\dagger}$ and M. A. Jeffris ${ }^{\ddagger}$ \\ Center for Research in Scientific Computation \\ North Carolina State University \\ Raleigh, NC 27695-8205
}

\begin{abstract}
In this paper, we present some numerical results of the determination of flow parameters in a groundwater model. The data used in this parameter estimation is from the MADE experiments conducted on Columbus Air Force Base. Our results are based on least squares cost functional with a finite difference scheme used to solve the flow equation.
\end{abstract}

\section{Introduction}

The modeling of groundwater flow is a crucial step in any quantitative analysis of subsurface contamination. From site characterization to remediation, flow models provide important decision-making tools. The main difficulty in applying such flow models is the determination of flow parameters such as hydraulic conductivity, which may vary by several orders of magnitude over a particular field site. In this paper, we examine a least squares parameter estimation technique which allows models to be calibrated to field data.

The approach we take here begins with a simple model of flow in a saturated porous medium. The coefficient and boundary values must be estimated as functions, from discrete observations made in the interior of the region of interest. A

*Research supported in part by AFOSR grant F49620-93-1-0355 and by a Department of Education GAANN Fellowship, grant P200A40730

${ }^{\dagger}$ Research supported in part by AFOSR grant F49620-93-1-0153.

${ }^{\ddagger}$ Research supported in part by AFOSR grant F49620-93-1-0153. simple finite difference scheme, containing flux balance conditions over conductivity discontinuities, is used to approximate the flow equation. Piecewise constant functions are used for the conductivities, and piecewise linear functions are used for the boundary values. For conductivity estimation, we use total variation constraints in the least squares fit-to-data criterion.

The basic model of steady state flow of groundwater in saturated soil is the elliptic equation

$$
\nabla \cdot(k \nabla h)=0 \quad x \in \Omega,
$$

where $k$ is the hydraulic conductivity, $h$ is the hydraulic head, and $\Omega$ is a subsurface region (in $\mathbb{R}^{2}$ or $\mathbb{R}^{3}$ ) of interest. The equation (1) is based on Darcy's empirical law, which states that groundwater velocity is proportional to the hydraulic gradient

$$
v=-k \nabla h,
$$

which, when combined with incompressibility and conservation of mass arguments, leads to (1). The references Freeze and Cherry (1975) and Bear (1972) provide a detailed discussion of the Darcy model.

For site characterization and remediation analyses, the groundwater velocity must be computed. In order to determine the velocity, one must solve (1), for which we need to know the function $k$ and the boundary values of $h$. The inverse problem of interest here is the determination of the function $k$ and $g=\left.h\right|_{\partial \Omega}$ from pointwise or distributed observations. In general one expects $k$ to be discontinuous (due, e.g., to layering in the subsurface), and it is not known a priori where these discontinuities lie. Thus, in the inverse problem we must search for $k$ 's from a very general class of functions. 
Typical field experiments yield data points $\left\{\hat{h}_{i}\right\}_{i=1}^{n_{1}}$ and $\left\{\hat{k}_{j}\right\}_{j=1}^{n_{2}}$, which correspond to observations of $h$ at the points $x_{i}$ and of $\left(1 /\left|\omega_{j}\right|\right) \int_{\omega_{j}} k(x) d x$ in which $\omega_{j}$ denotes a "small" set in $\Omega$ and $|A|$ denotes the Lebesgue measure of $A$ (the averaging nature of this observation operator is based on the borehole flowmeter measurement device: see Rehfeldt, et. al., 1992a, 1992b).

To determine parameters based on these data, we define the least squares cost functional

$$
\begin{aligned}
J(k, g) & =\sum_{i=1}^{n_{1}}\left|h\left(x_{i} ; k, g\right)-\hat{h}_{i}\right|^{2} \\
& +\beta \sum_{j=1}^{n_{2}}\left|\frac{1}{\left|\omega_{j}\right|} \int_{\omega_{j}} k(x) d x-\hat{k}_{j}\right|^{2}
\end{aligned}
$$

which is to be minimized over some appropriate collection $\mathcal{K} \times \mathcal{G}$ of functions $k$ and $g$. The number $\beta$ is a weighting parameter which must be used due to differences (by orders of magnitude) in the values of $h$ and $k$. In the next section, we examine theoretical aspects of this estimation problem.

\section{Theoretical considerations}

In this section we recall some important results from analysis and approximation in elliptic partial differential equations such as (1). We denote by $H^{k}=H^{k}(\Omega)$ and $H_{0}^{k}=H_{0}^{k}(\Omega)$ the usual Sobolev spaces of weakly differentiable functions. Throughout, we assume that $\Omega$ is a bounded open subset of $\mathbb{R}^{m}$. The notation $\left.f\right|_{\partial \Omega}$ is used in general to denote the trace of an $H^{1}$ function. We also use the notation $H^{s}(\partial \Omega)$ to denote the fractional order (i.e., $s>0$ real) trace spaces of Lions (see, e.g., Wloka, 1987).

To solve the differential equation (1) subject to the boundary condition $\left.h\right|_{\partial \Omega}=g$, we seek a function $h \in H^{1}$ of the form $h=u+G$, where $G \in H^{1}$ satisfies $\left.G\right|_{\partial \Omega}=g$ and where $u \in H_{0}^{1}$ satisfies

$$
\nabla \cdot(k \nabla u-k \nabla G)=0
$$

in $\Omega$. It is well known (see, e.g., Wloka, 1987) that there is a bounded linear extension operator $Z: H^{1 / 2}(\partial \Omega) \rightarrow H^{1}$ such that the trace of $Z f$ is $f$, for each $f \in H^{1 / 2}(\partial \Omega)$. The determination of $h$ is then based on solving (4) for $u$. Mulitplying in
(4) by a test function $\phi$ and integrating by parts, we have

$$
\sigma_{k}(u, \phi)=F_{g, k}(\phi),
$$

in which the bilinear form $\sigma_{k}: H_{0}^{1} \times H_{0}^{1} \rightarrow \mathbb{R}$ is given by

$$
\sigma_{k}(\phi, \psi)=\int_{\Omega} k(x) \nabla \phi(x) \cdot \nabla \psi(x) d x,
$$

and the bounded linear functional $F_{g, k}: H_{0}^{1} \rightarrow \mathbb{R}$ is given by $F_{g, k}(\phi)=\int_{\Omega} k(x) \nabla G(x) \cdot \nabla \phi(x) d x$, in which $G=Z(g) \in H^{1}$. We summarize the application of the Lax-Milgram theorem and the weak maximum principle in the following theorem (see Treves, 1975, or Wloka, 1987, for details of the proof).

Theorem 1 If the domain $\Omega$ is a bounded open set with Lipschitz continuous boundary $\partial \Omega$, if $g \in H^{1 / 2}(\partial \Omega)$ and $k \in L^{\infty}(\Omega)$, and if there exist positive numbers $\alpha, \beta$ such that $\alpha \leq k(x) \leq \beta$ a.e., then the equation (5) has a unique solution $u \in H_{0}^{1}$, and the equation (1) has a unique solution $h \in H^{1}$ satisfying $\left.h\right|_{\partial \Omega}=g$.

We remark that in the proof of this theorem, which is essentially contained in the references Treves, (1975), and Wloka (1987), the coercivity and boundedness of $\sigma$ are crucial: $\sigma$ satisfies

$$
\begin{array}{r}
\alpha\|\phi\|_{H_{0}^{1}}^{2} \leq \sigma_{k}(\phi, \phi), \\
\left|\sigma_{k}(\phi, \psi)\right| \leq \beta\|\phi\|_{H_{0}^{1}}\|\psi\|_{H_{0}^{1}}
\end{array}
$$

in which $\|\phi\|_{H_{0}^{1}}^{2}=\int_{\Omega}|\nabla \phi(x)|^{2} d x$ is the norm on $H_{0}^{1}(\Omega)$.

Another crucial estimate involves the sup norm of the solution. Below we state a form of Harnack's inequality, which may be found in full generality in Gilbarg and Trudinger (1983). First, we need some notation. If $\Omega_{0}$ and $\Omega_{1}$ are open sets, we write $\Omega_{0} \subset \subset \Omega_{1}$ if the closure $\bar{\Omega}_{0}$ is a compact subset of $\Omega_{1}$.

Theorem 2 (Harnack's inequality) If $\Omega$ is a bounded open set and if $\omega \subset \subset \Omega$, then there exist constants $C, \lambda>0$ such that

$$
\|u\|_{C^{\lambda}(\bar{\omega})} \leq C\|u\|_{L^{2}(\Omega)}
$$

for each pair $u \in H^{1}, k \in L^{\infty}(\Omega)$ such that $0<$ $\alpha \leq k(x) \leq \beta$, such that $\nabla \cdot(k \nabla u)=0$, where $C^{\lambda}$ denotes as usual the space of Hölder continuous functions of exponent $\lambda$. 
As noted in Gilbarg and Trudinger (1983), the particular choice of the constants $C$ and $\lambda$ depends only on $\beta / \alpha$ and $\operatorname{dist}(\partial \Omega, \omega)$, and not on the particular coefficient function $k \in L^{\infty}$ or solution $u \in H^{1}$. It is sup norm regularity that enables us to handle pointwise observation operators in the inverse problem, as we shall see below.

For the parameter estimation problem, we let

$$
\begin{aligned}
\mathcal{K}= & \left\{\phi \in L^{\infty}(\Omega): 0<\alpha \leq \phi(x) \leq \beta,\right. \\
& \text { a.e. } x \in \Omega, T V(\phi)<\rho\}
\end{aligned}
$$

where the total variation $T V(\phi)$ is defined as

$$
\begin{aligned}
T V(\phi)= & \sup \left\{\int_{\Omega} \phi(x)(\nabla \cdot F)(x) d x:\right. \\
& \left.F \in C^{1}\left(\bar{\Omega} ; \mathbb{R}^{m}\right), \sup _{x \in \Omega}|F(x)| \leq 1\right\},
\end{aligned}
$$

where $\Omega \subset \mathbb{R}^{m}$ with $m \leq 3$. In Gutman (1990) it is proved that $\mathcal{K}$ is a compact subset of $L^{1}(\Omega)$, when $\Omega$ is a bounded open set in $\mathbb{R}^{m}$. For the boundary conditions, we fix a positive constant $\Delta$, and we set

$$
\mathcal{G}=\left\{\phi \in H^{1}(\partial \Omega):\|\phi\|_{H^{1}(\partial \Omega)}^{2} \leq \Delta\right\},
$$

which is compact in $H^{\frac{1}{2}}(\partial \Omega)$ if the boundary $\partial \Omega$ is Lipschitz continuous (see, e.g., Wloka, 1987). The total variation constraints allow for discontinuous conductivity functions: this flexibility is lacking in most other types of compactness constraints.

Theorem 3 Let $\left(g_{n}\right)$ be a sequence of functions in $\mathcal{G}$ such that $g_{n} \longrightarrow g$ in $\mathcal{G}$. Let $\left(k_{n}\right)$ be a sequence of functions in $\mathcal{K}$ such that $k_{n} \rightarrow k \in \mathcal{K}$ in the $L^{1}$-norm. For each $n$, define $u_{n} \in H_{0}^{1}(\Omega)$ to be the Lax-Milgram solution of (6) using $k_{n}$ and $G_{n}$, where $G_{n}=Z\left(g_{n}\right) \in H^{1}(\Omega)$ is the extension of $g_{n}$. Then, $u_{n} \rightarrow u$ in $H^{1}(\Omega)$ where $u$ is the Lax-Milgram solution using $k$ and $G$, and $G=Z(g) \in H^{1}(\Omega)$ is the extension of $g$. Moreover, we have that for each open set $\omega_{0}$ with $\omega_{0} \subset \subset \Omega, h_{n}\left(\cdot ; g_{n}, k_{n}\right) \rightarrow h(\cdot ; g, k)$ uniformly on $\omega_{0}$, where $h_{n}=u_{n}+G_{n}$, and $h=u+G$.

The proof of this result, contained in Fitzpatrick and Jeffris (1994), relies in a crucial way on Harnack's inequality as stated above.

In general, there are two main obstacles to implementation of this approach: the function $h$ must be computed numerically and the set $\mathcal{K} \times \mathcal{G}$ must be approximated by a finite dimensional set. The paper of Fitzpatrick and Jeffris (1994), contains analysis of this least squares problem in terms of existence of minimizers and convergence of numerical approximations to the least squares cost. The results contained therein pertain to standard finite element methods for solving the flow equation, together with rather smooth approximations to the conductivity parameter. In the present work we use a simple finite difference scheme with piecewise constant conductivity functions. The scheme is easy to implement and captures the the behavior of the conductivity rather well. We do not, however, have a complete convergence analysis, and this matter is a topic of our current research. In the following section we give the details of our numerical approach for these approximations.

\section{Numerical Implementation}

Our numerical scheme begins with the simplifying assumption that the flow is two dimensional. While it would seem a natural assumption for a shallow aquifer, in which the horizontal dimensions are 30 to 50 times the vertical dimension, we shall see in the computations below that this assumption may not be valid. However, in order to develop some insight into the flow at a particular site, using two dimensional slices is an instructive first step. Thus, we assume that the region $\Omega$ of interest is a rectangle: $\Omega=(a, b) \times(c, d)$. We subdivide this rectangle into $m_{1} * m_{2}$ subrectangles of equal lengths and widths, $(b-a) / m_{1}$ and $(d-c) / m_{2}$, respectively. We assume that the conductivity $k$ is constant on these subdomains.

The next step is to approximate the differential equation with a difference equation. We divide the region $\Omega$ into a grid of size $\ell_{1} * \ell_{2}$, with $\ell_{i}=s_{i} * m_{i}+1$ for $i=1,2$ where the $s_{i}$ 's are integers. This assures that the finite difference grid is aligned with the conductivity grid. Solving the differential equation from the boundary values in the forward problem produces a system of $\left(\ell_{1}-2\right) *\left(\ell_{2}-2\right)$ equations and unknowns. The difference equation we use is the following. If the index pair $(i, j)$ corresponds to a grid point in the 
interior of a conductivity subregion, we have

$$
\begin{aligned}
& \frac{h_{i+1, j}-2 h_{i, j}+h_{i-1, j}}{\Delta x} \\
+ & \frac{h_{i, j+1}-2 h_{i, j}+h_{i, j-1}}{\Delta y}=0,
\end{aligned}
$$

in which $\Delta x$ and $\Delta y$ denote the spatial discretization length and width for the finite difference grid, and $h_{i, j}$ is the computed value of the hydraulic head at the $(i, j)$ grid point. Note that the conductivity is constant in the subdomain containing all the grid points, so it may be divided out of the equation. If the point $(i, j)$ lies on a conductivity subdomain boundary which is parallel to the $x$ axis, we use the flux balance equation

$$
\frac{k_{a}\left(h_{i, j+1}-h_{i, j}\right)}{\Delta y}=\frac{k_{b}\left(h_{i, j}-h_{i, j-1}\right)}{\Delta y},
$$

in which $k_{a}$ denotes the value of conductivity above the boundary and $k_{b}$ denotes the value below. Likewise, if the point $(i, j)$ is on a boundary parallel to the $y$ axis, we use

$$
\frac{k_{l}\left(h_{i, j}-h_{i-1, j}\right)}{\Delta x}=\frac{k_{r}\left(h_{i+1, j}-h_{i, j}\right)}{\Delta x},
$$

where $k_{l}$ and $k_{r}$ denote the conductivity values from the subdomains to the left and right, respectively, of the boundary. Points on the "corners" of the subdomains appear in none of the other equations, so it is unnecessary to compute these values (we take the average of the four nearest neighbor points for the purposes of generating graphs below).

To solve the forward problem for a particular choice of $k$ and $g$ values, we use the GMRES algorithm (see Saad and Schulz, 1992) to solve this system of $\left(\ell_{1}-2\right) *\left(\ell_{2}-2\right)$ linear equations. This forward solver is then coupled with the FORTRAN optimization routine IFFCO (see Gilmore, 1993, and Gilmore and Kelley, 1992), which is a quasi-Newton iteration coupled with implicit filtering (through careful differencing) for avoiding local minima (Our original efforts using LMDIF1, a FORTRAN implementation of the LevenbergMarquardt algorithm for nonlinear least squares problems, available through netlib, encountered some difficulties due to local minima).

The upper and lower bound constraints are implemented directly in IFFCO. The integral and total variation constraints on $g$ and $k$, however, are more difficult to implement directly and must be incorporated into the cost functional as penalty terms. The cost functional which we use is given by

$$
\begin{aligned}
& J(k, g)=\sum_{i=1}^{n_{1}}\left|h\left(\vec{x}_{i}, k, g\right)-\hat{h}_{i}\right|^{2} \\
& +\beta_{1} \int_{\partial \Omega}\left|g^{\prime}\right|^{2} d s \\
& +\beta_{2} \sum_{(i, j)} \sum_{(l, m) \in \mathcal{N}(i, j)} \sqrt{\alpha+\left|k_{i, j}-k_{l, m}\right|^{2}}, \\
& +\beta_{3} \sum_{j=1}^{n_{2}}\left|k\left(\vec{y}_{j}\right)-\hat{k}_{j}\right|^{2},
\end{aligned}
$$

in which $\beta_{1}, \beta_{2}$, and $\beta_{3}$ are weighting parameters that must be chosen, and $\alpha$ is a smoothing parameter. The final term in the cost functional approximates the total variation of the function $k$. The notation $\mathcal{N}(i, j)$ denotes the $k$ subdomain indices having sides adjacent to the $(i, j)$ subdomain. Since the approximation takes $k$ to be a piecewise constant function, we use the notation $k_{i}(i, j)$ and $k(x)$ for values of $k$, the former being the value on the $(i, j)$ subdomain. The final piece of notation is the point $\vec{y}_{j}$, denoting the center of the region $\omega_{j}$ in which the borehole flowmeter operates. We now proceed to an examination of the MADE data.

\section{Conductivity Estimates for the MADE site}

The papers of Rehfeldt et. al. (1992a, 1992b) and Boggs et. al. (1993) describe in detail the MADE experiments as well as some rather extensive data analysis. The site over which the measurements were taken is roughly $400 \times 600$ feet in horizontal area and about 30 feet in depth. The site consists of a very heterogeneous mixture of soil types on the Columbus Air Force Base is Mississippi. Approximately 60 borehole flowmeter measurements from the MADE- 1 experiment are used in our analysis, with about 10 head measurements taken from multilevel sampling well.

We see in Figures 1, 2, and 3 the head, conductivity, and velocity field using vertically averaged conductivity data. In Figures 4, 5, and 6, we see the estimates using the conductivity data 
in the horizontal slab between 60 and 61 meters above sea level. The dramatic difference in velocity fields indicates the possible need for three dimensional computations, which we are currently pursuing. These computations used $10 \times 10 \mathrm{sub}-$ domains for the conductivity, with $51 \times 51$ grid points for the head computations. Three linear spline functions per side were used to estimate the boundary values at the edge of the rectangle. After many sensitivity studies, values of $\alpha, \beta_{1}, \beta_{2}$, and $\beta_{3}$ were chosen at $10^{-2}, 8,2$, and 8 for our computations.

In Figures 1 and 2, the asterisks indicate the vertically averaged data from the MADE experiments, while in Figures 4 and 5, the asterisks indicate the actual data in the 60-61 meters above sea level vertical range. The velocity plots contain contour lines of the hydraulic head, subdomain boundaries for the conductivity estimates, and circles to indicate the borehole flowmeter locations (where conductivity data are taken). We remark that the heterogeneous nature of the medium is particularly evident in the velocity plots. In a homogeneous medium one would expect "closeness" of level curves of head to indicate high velocity regions. However, the variation in the conductivity is also a factor, one which seems in this study to be the driving influence.

\section{Acknowledgements}

The authors would like to thank Dr. T. B. Stauffer and Dr. S. Kang, AL/EQ, Tyndall AFB, for providing the MADE experiment data, and for their helpful comments concerning the experiment and the data collection and analysis.

\section{References}

H.T. Banks and K. Kunisch, 1989. "Estimation Techniques for Distributed Parameter Systems," Birkhäuser, Boston.

J. Bear, 1972. "Dynamics of Fluids in Porous Media," Elsevier, New York.

J. M. Boggs, L. M. Beard, S. E. Long, M. P. McGee, W. G. MacIntyre, C. P. Antworth, and T. B. Stauffer, 1993. Database for the Second Macrodispersion Experiment
(MADE-2), Electric Power Research Institute Technical Report TR-102072, EPRI, Palo Alto.

B. G. Fitzpatrick and M. A. Jeffris, 1994. "Parameter Estimation in Groundwater Flow Models with Distributed and Pointwise Observations," Technical Report CRSCTR94-17, Center for Research in Scientific Computation, North Carolina State University, Raleigh.

R. A. Freeze and J. Cherry, 1979. Groundwater, Prentice-Hall Englewood Cliffs.

S. Gutman, 1990. Identification of Discontinuous Parameters in Flow Equations, SIAM Journal on Control and Optimization $\mathbf{2 8}$ (5), pp. 1049-1060.

D. Gilbarg and N. S. Trudinger, 1983. Elliptic Partial Differential Equations of Second Order, Springer-Verlag, Berlin.

P. Gilmore, 1993. "IFFCO: Implicit Filtering for Constrained Optimization, Users' Guide, Technical Report CRSC-TR93-7, Center for Research in Scientific Computation, North Carolina State University, Raleigh.

P. Gilmore and C. T. Kelley, 1992. "An Implicit Filtering Algorithm for Optimization of Functions with Many Local Minima," Technical Report CRSC-TR92-22, Center for Research in Scientific Computation, North Carolina State University, Raleigh; to appear in SIAM Journal on Optimization.

Y. Saad and M. H. Schultz, 1986. "GMRES: A Generalized Minimal Residual Algorithm for Solving Nonsymmetric Linear Systems", SIAM Journal on Scientific and Statistical Computing, 7 (3), pp. 856-869.

F. Treves, 1975. Basic Linear Partial Differential Equations, Academic Press, Orlando.

K. R. Rehfeldt, L. W. Gelhar, S. C. Young, E. E. Adams, L. M. Beard, and J. M. Boggs, 1992a. "Fieid Study of Dispersion in a Heterogeneous Aquifer: 1. Oveview and Site Description," Water Resources Research, 28 (12): 3281-3291.

K. R. Rehfeldt, J. M. Boggs, and L. W. Gelhar, 1992b. Field Study of Dispersion in a Heterogenenous Aquifer: 3. Geostatistical 
Analysis of Hydraulic Conductivity, Water Resources Research, 28 (12), pp. 33093324.

Wloka, J., 1987 Partial Differential Equations, Cambridge University, Cambridge.

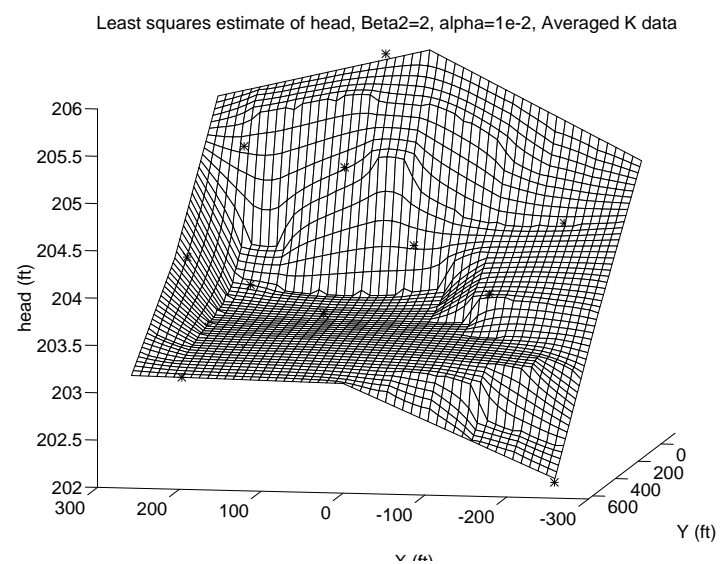

Figure 1: Hydraulic head estimate using vertically averaged data

Least squares estimate of Conductivity, Beta2 $=2$, alpha $=1 \mathrm{e}-2$, Averaged $\mathrm{K}$ data

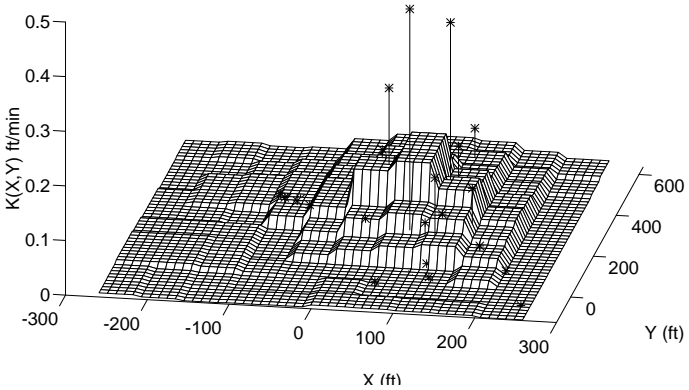

Figure 2: Hydraulic conductivity estimate using vertically averaged data

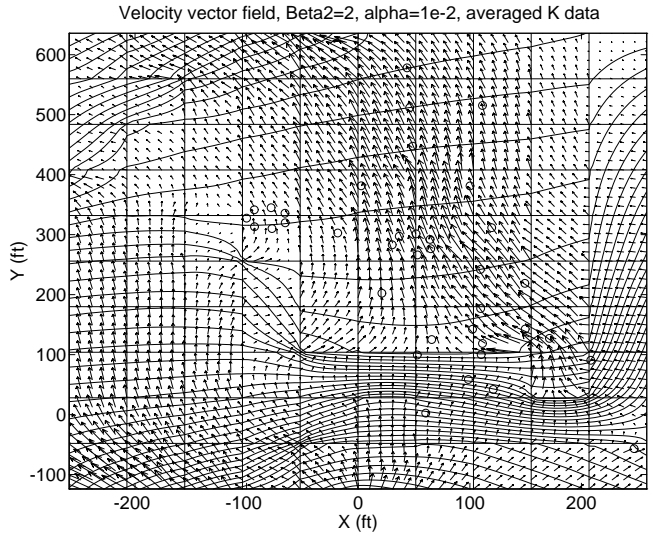

Figure 3: Velocity field estimate using vertically averaged data

Least squares estimate of head, Beta2 $=2$, alpha $=1 \mathrm{e}-2, \mathrm{~K}$ data with $60<=\mathrm{Z}<=61$

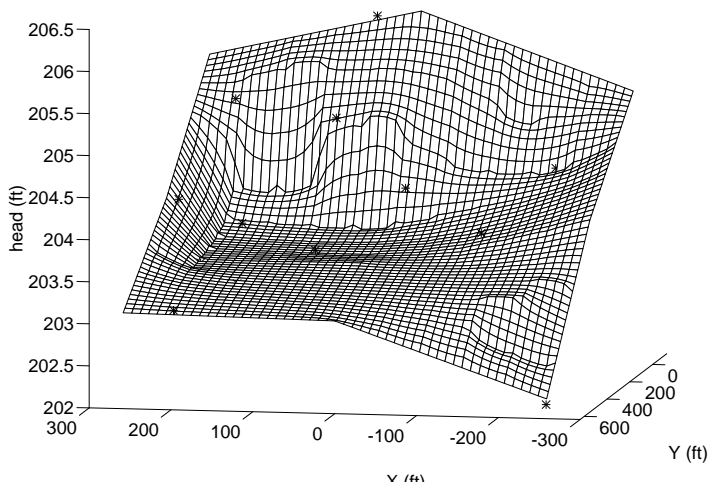

Figure 4: Hydraulic head estimate using vertical data, 60-61 meters

Least squares est. of $K(x, y)$, Beta2 $=2$, alpha $=1 e-2, K$ data with $60<=z<=61$

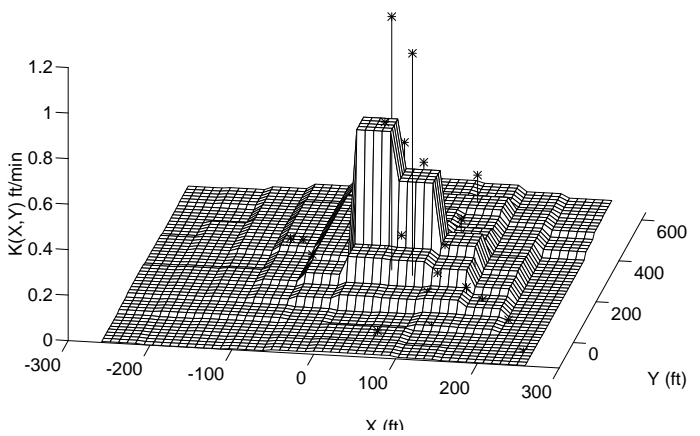

Figure 5: Hydraulic conductivity estimate using vertical data, 60-61 meters 


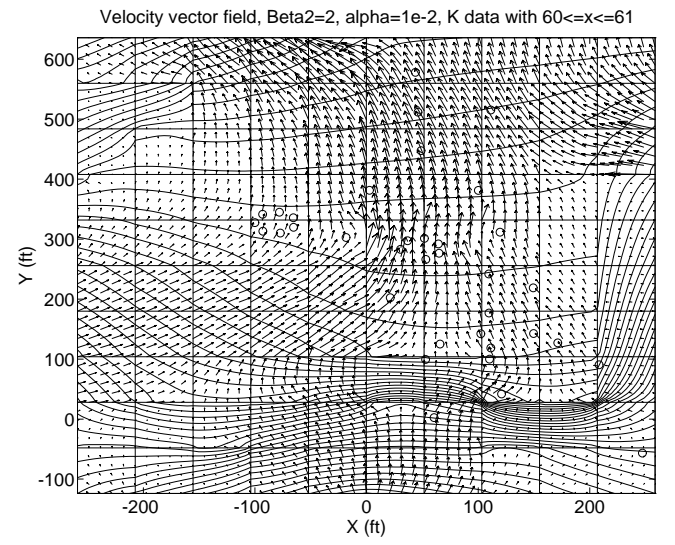

Figure 6: Velocity field estimate using vertical data, 60-61 meters 